\title{
Descripción morfométrica y faneroptica de la cabra "Chusca lojana" del bosque seco del Sur del Ecuador
}

\author{
Aguirre, E.L. ${ }^{1}$, Quezada, M. ${ }^{1}$, Maza, T. ${ }^{1}$, Albito, O. ${ }^{1}$, Armijos D.R. ${ }^{1}$, Flores, A. ${ }^{2}$ y Camacho, O².
}

'Carrera de Medicina Veterinaria y Zootecnia. Facultad Agropecuaria. Universidad Nacional de Loja. Loja. Ecuador.

${ }^{2}$ Tesistas Medicina Veterinaria y Zootecnia. Universidad Nacional de Loja. Loja. Ecuador.

\section{PaLABRAS CLAVE}

Cabra criolla.

Caracterización.

Dimorfismo sexual.

Fenotipo.

Provincia de Loja.

\section{RESUMEN}

En la Región Sur del Ecuador se encuentra la zona del bosque seco que abarca desde los 100 metros sobre el nivel del mar (m.s.n.m) hasta los 1200 m.s.n.m, lugar donde se ha adaptado la cabra criolla llamada "Chusca lojana". El presente trabajo tuvo como objetivo la caracterización fenotípica de este recurso zoogenético, permitiendo disponer de un patrón o estándar racial con fines de identificación, estudio, selección y mejoramiento de esta población. La investigación comprendió una muestra de 163 cabras adultas manejadas en ambiente extensivo, considerando 21 medidas morfométricas y 10 características fanerópticas; para el análisis morfométrico se aplicó una estadística no paramétrica con el Test de Kruskal-Wallis considerando como variables el sexo y los biotipos. Los resultados permitieron identificar 4 biotipos, que de acuerdo a ciertas características peculiares que presentan a nivel de cabeza y orejas se las ha identificado como: Chusca "oreja corta y doblada", Chusca "oreja de león", Chusca oreja "torneada" y Chusca "muca"; en el aspecto faneróptico presentan cuernos opistoceros $(58 \%)$ y ortoceros (37\%), siendo un $22 \%$ acornes (biotipo Chusca muca), poseen barba y mamelas el 26 y $29 \%$, respectivamente, presentando estas dos características un dimorfismo en favor de los machos; la mayoría de esta población presenta un perfil de rostro recto $(67 \%)$ y subcóncavo $(28 \%)$; la forma de la ubre es globosa $(64 \%)$, el color de pezones $(93 \%)$ y cascos $(87 \%)$ es pigmentado; los machos presentan testículos pendulosos $(70 \%)$ y una característica peculiar es que poseen un escroto dividido en dos sacos $(40 \%)$. En conclusión, la cabra Chusca es un animal con una tendencia productiva más a leche que a carne, destacando su capacidad torácica que le permite recorrer con facilidad por la topografía irregular de la zona en busca de alimento.

\section{Morpho-faneroptic description of the goat "Chusca lojana" of the dry forest of the Southern of Ecuador}

\section{SUMMARY}

\section{AdDITIONAL KEYWORDS}

Characterization.

Creole goat.

Phenotype.

Province of Loja.

Sexual dimorphism.

\section{INFORMATION}

\section{Cronología del aríículo.}

Recibido/Received: 07.10.2020

Aceptado/Accepted: 20.11 .2020

On-line: 15.04 .2021

Correspondencia a los autores/Contact e-mail:

edgar.aguirre@unl.edu.ec
In the Southern Region of Ecuador a dry forest area that covers from 100 meters above sea level (m.a.s.l) to 1200 m.a.s.l, was find place where the Creole goat called "Chusca lojana" has been adapted. The present work aimed at the phenotypic characterization of this animal genetic resource, specifying a racial pattern or standard with fines for identification, study, selection and improvement of this population. The research included a sample of 163 adult goats managed in an extensive environment, specific 21 morphometric measures and 10 pheno-optical characteristics; for the morphometric analysis a nonparametric statistic was applied with the Kruskal-Wallis Test as variable variables of sex and biotypes. The results allowed to identify 4 biotypes that according to certain peculiar characteristics that have a level of head and ears have been identified as: Chusca "short and bent ear", Chusca "lion ear", Chusca ear "turned" and Chusca "muca "; in the phanero-optic aspect, they have opistocer (58\%) and orthoceros (37\%) horns, being $22 \%$ acorns (Chusca muca biotype), 26 and $29 \%$ have a beard and mamelas respectively, presenting these two characteristics a dimorphism in favor of males, the majority of this population has a straight $(67 \%)$ and subconcave $(28 \%)$ face profile; the udder shape is globose $(64 \%)$, the color of nipples $(93 \%)$ and helmets (87\%) is pigmented; males have pendulous testicles $(70 \%)$ and a peculiar characteristic is that they have a scrotum divided into two sacs $(40 \%)$. In conclusion, the Chusca goat is an animal with a productive tendency more to milk than to meat, highlighting its thoracic capacity that allows it to move easily through the irregular topography of the area in search of food.

\section{INTRODUCCIÓN}

La cabra (Capra aegagrus hircus), tradicionalmente tiene una fuerte influencia socio-económica en la población rural asentada en los sectores menos favorecidos en todo el mundo, pues la ganadería caprina constituye una importante fuente de proteína al aprovechar los diferentes recursos naturales de baja calidad 
existentes en esas zonas y que otras especies animales no los utilizan (Dubeuf et al. 2004). La Región Sur del Ecuador, debido a su relieve montañoso se caracteriza por poseer una abrupta y empinada topografía que ha dado lugar a diferentes pisos altitudinales con microclimas y suelos diversos, por tanto especies de animales y vegetales adaptadas a cada uno de estas microrregiones que requieren periodos largos de adaptación, ocasionan que los individuos tarden generaciones en desarrollar y manifestar su potencial genético-productivo (Falconer \& Mackay 1996). Por ello se puede entender, que en esta región existe una variabilidad genética que no se la aprovechado y proporcionado la importancia para su selección, difusión y conservación, más bien lo que ha ocurrido es la implementación de programas de mejoramiento genético en las distintas especies, que ha consistido en la introducción de razas y cruzamientos sin control que han ido desvalorizando y diluyendo aquel material genético existente in situ.

En este ambiente se encuentra la zona del bosque seco que abarca diferentes pisos altitudinales desde los 100 m.s.n.m, en Zapotillo hasta los 1200 m.s.n.m, en la zona de Gonzanamá, Calvas y Paltas, se puede entender que en este ecosistema de escasa producción de biomasa forrajera, el animal que mejor se ha adaptado es la cabra criolla llamada "Chusca", animal que al igual que otras especies nativas o criollas se encuentran cada día disminuyendo su número efectivo de individuos, situación corroborada por Bélanger (2019), al manifestar que las amenazas a la diversidad de plantas y animales y la proporción de razas en peligro de extinción cada día se incrementan.

Estudios realizados en esta población no han sido desarrollados en ningún campo, por lo que no se dispone de información técnica que permita tomar decisiones referentes a su cuidado, manejo y conservación, generando con ello un impacto positivo en las condiciones socioeconómicas y ambientales del capricultor del bosque seco de la Provincia de Loja. Por tal razón se justifica el presente trabajo de caracterización fenotípica de este recurso zoogenético, pues, se logrará con ello disponer de un patrón o estándar racial que permita en un futuro inmediato su estudio, mejoramiento y multiplicación.

\section{MATERIAL Y MÉTODOS}

La investigación comprendió una muestra de 163 cabras adultas (153 hembras y 10 machos) manejadas en ambiente extensivo en la zona del bosque seco de la Provincia de Loja al sur del Ecuador, que abarca diferentes pisos altitudinales desde los 100 m.s.n.m, en Zapotillo hasta los 1.200 m.s.n.m, en la zona de Gonzanamá, Calvas y Paltas. Fueron considerados en este análisis el peso corporal adulto y 21 medidas morfométricas: longitud corporal (LC), longitud de cara (Lca), longitud de oreja (LO), longitud de caña anterior (LcñA) y posterior (LcñP), longitud de grupa (LG), longitud de ubre (LU) y pezones (LP), longitud de cuernos (Lcu), ancho de pecho (AP), ancho de grupa anterior (AGa) y posterior (AGp), ancho de cara (AC), ancho de oreja (AO), perímetro torácico (PT) y abdominal (PA), perímetro de caña $(\mathrm{PC})$, diámetro testicular (DT), altura a la cruz (ALC), altura a la grupa (ALG) y altura dorsoesternal (ADE); también se analizaron 10 características fanerópticas: color de la capa, presencia y forma de cuernos, presencia de mamelas y/o barba, perfil del rostro, forma de ubre y testículos, color de pezones y pezuñas. Para el análisis morfométrico se aplicó una estadística no paramétrica con el Test de Kruskal-Wallis (Carné et al. 2007) y para las características fanerópticas se aplicó una estadística descriptiva (Hernandez et al. 2002, Sunder et al. 2018), considerando en ambos análisis como variables el sexo y los biotipos.

\section{RESULTADOS}

En el presente estudio se determinó, que la cabra Chusca alcanza un peso a la madurez: $q$ de $40 \pm 1.3 \mathrm{~kg}$ a los 48 meses y $\precsim$ de $62.5 \pm 4 \mathrm{~kg}$ a los 50 meses. En relación a las medidas morfométricas de este animal (Tabla I), se observó que se trata de una población homogénea en cuanto a su morfoestructura como lo demuestra la baja variabilidad (CV) que no supera el $15 \%$ en las 21 medidas analizadas, características como longitud de cuernos y longitud de orejas, presentaron un CV de 10.6 y 9.2, respectivamente, permitiendo de acuerdo al criterio de Manson (1981), agrupar a esta población en 4 biotipos por la presencia o no de cuernos y por la forma y tamaño de las orejas, identificando los biotipos en Chusca "Muca" (ausencia de cuernos), Chusca "oreja de león" (oreja pequeña), Chusca oreja "torneada" (pabellón de la oreja doblado) y Chusca oreja "corta y doblada" (Figura 1).

Al analizar medidas morfométricas de importancia económica, presenta una longitud corporal (LC) de $72.2 \pm 1.1 \mathrm{~cm}$; una alzada a la cruz (ALC) y a la grupa (ALG) de $66.8 \pm 0.8 \mathrm{~cm}$ y $67.3 \pm 0.6 \mathrm{~cm}$, respectivamente, dándole una línea dorso-lumbar con una ligera lordosis; el perímetro torácico (PT) $\left(q: 77.6, \bigcirc^{\Uparrow}: 88.8 \mathrm{~cm}\right)$; ancho de pecho (AP) (: $15.6, \hat{\jmath}: 19.7 \mathrm{~cm})$ que denota la fortaleza del animal para desplazarse con facilidad por topografías accidentadas; la altura dorso-esternal (ADE) que representa la profundidad del animal en esta cabra es de $30.2 \pm 0.7 \mathrm{~cm}$.
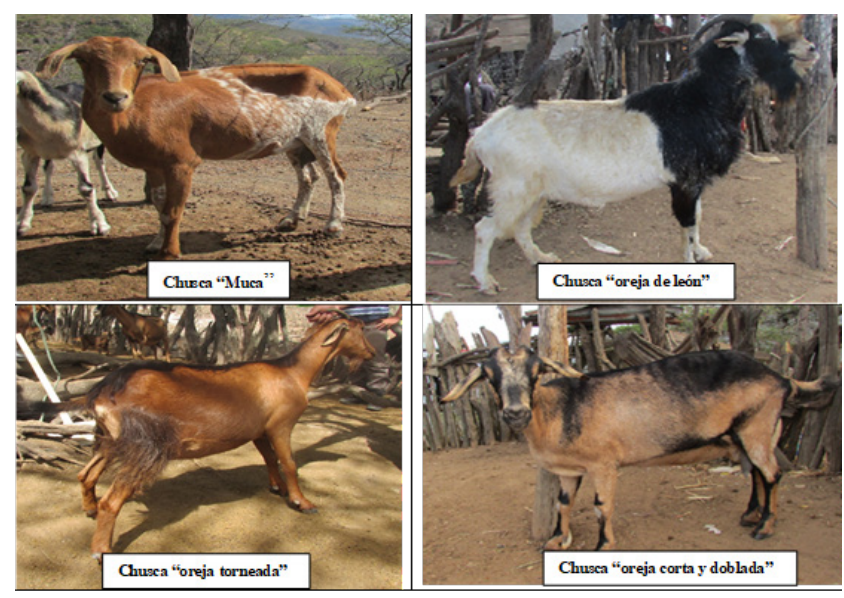

Figura 1. Biotipos identificados en la cabra Chusca del bosque seco del sur del Ecuador (Biotypes of the Chusca lojana goat from dry forest of Southern of Ecuador). 
Tabla I. Características morfométricas $(\mathrm{cm})$ de la cabra “Chusca lojana" (Morphometric characteristics of the "Chusca lojana" goat).

\begin{tabular}{|c|c|c|c|c|c|c|c|c|c|c|c|c|c|c|c|c|c|c|c|c|c|}
\hline \multirow{3}{*}{ Biotipo } & \multirow{3}{*}{ LC } & \multirow{3}{*}{ LCa } & \multirow{2}{*}{\multicolumn{3}{|c|}{ Lcñ }} & \multirow{3}{*}{ LG } & \multirow{3}{*}{ LU } & \multirow{3}{*}{ LP } & \multirow{3}{*}{ LCu } & \multirow{3}{*}{ AP } & \multirow{2}{*}{\multicolumn{2}{|c|}{$A G$}} & \multirow{3}{*}{ AC } & \multirow{3}{*}{$\mathrm{AO}$} & \multirow{3}{*}{ PT } & \multirow{3}{*}{ PA } & \multirow{3}{*}{ PC } & \multirow{3}{*}{ DT } & \multirow{3}{*}{ ALC } & \multirow{3}{*}{ ALG } & \multirow{3}{*}{ ADE } \\
\hline & & & & & & & & & & & & & & & & & & & & & \\
\hline & & & & LcñA & LcñP & & & & & & AGa & AGp & & & & & & & & & \\
\hline$C D$ & 70.7 & 20.2 & $12.8^{\mathrm{a}}$ & 6.6 & 7.3 & 20.9 & 17.3 & $3.3^{\mathrm{a}}$ & $13.4^{\mathrm{b}}$ & 15.5 & 12.0 & 7.8 & 10.0 & 7.2 & 77.5 & 92.7 & 7.2 & 26.6 & 66.0 & 66.7 & 29.6 \\
\hline Mucos & 73.5 & 20.1 & $12.6^{\mathrm{a}}$ & 7.0 & 7.5 & 21.9 & 16.6 & 3.3 $3^{\mathrm{a}}$ & -- & 16.0 & 12.2 & 8.1 & 10.3 & 7.5 & 79.8 & 91.9 & 7.5 & 32.8 & 67.8 & 68.1 & 30.9 \\
\hline OL & 72.2 & 19.9 & $10.7^{b}$ & 6.9 & 7.5 & 21.3 & 17.1 & $2.7^{\mathrm{b}}$ & $16.1^{\circ}$ & 15.4 & 12.0 & 8.0 & 9.8 & 7.4 & 76.7 & 90.5 & 7.1 & 30.0 & 66.6 & 67.2 & 29.6 \\
\hline Torneada & 72.3 & 19.7 & $13.3^{\mathrm{a}}$ & 6.7 & 7.3 & 21.5 & 15.8 & $3.2^{\mathrm{a}}$ & $12.0^{\mathrm{a}}$ & 16.3 & 12.2 & 7.9 & 10.4 & 7.4 & 80.0 & 94.6 & 7.7 & 34.0 & 66.7 & 67.3 & 30.7 \\
\hline GENERAL & 72.2 & 20.0 & 12.3 & 6.8 & 7.4 & 21.4 & 16.7 & 3.1 & 13.8 & 15.8 & 12.1 & 8.0 & 10.1 & 7.4 & 78.5 & 92.4 & 7.4 & 30.9 & 66.8 & 67.3 & 30.2 \\
\hline $\mathrm{s}$ & 1.12 & 0.21 & 1.13 & 0.17 & 0.11 & 0.39 & 0.69 & 0.28 & 2.06 & 0.43 & 0.11 & 0.13 & 0.27 & 0.14 & 1.68 & 1.70 & 0.25 & 3.29 & 0.77 & 0.57 & 0.69 \\
\hline CV & 15 & 105 & 92 & 25 & 15 & 18 & 41 & 903 & 149 & 27 & 91 & 16 & 27 & 19 & 21 & 18 & 34 & 106 & 11 & 8 & 23 \\
\hline
\end{tabular}

LC: longitud corporal; Lca: longitud cara; LO: longitud oreja; LcñA: longitud caña anterior; LcñP: longitud caña posterior; LG: longitud grupa; LU: longitud ubre; LP: longitud pezones; Lcu: longitud cuernos; AP: ancho de pecho; AGa: ancho grupa anterior; AGp: ancho grupa posterior; AC: ancho de cara; AO: ancho de oreja; PT: perímetro torácico; PA: perímetro abdominal; PC: perímetro caña; DT: diámetro testicular; ALC: altura cruz; ALG: altura grupa; ADE: altura dorso-esternal. CD: biotipo oreja corta y doblada; OL: biotipo oreja de león; s: desviación estándar; CV: coeficiente de variación; *distintas letras en una misma columna: hay diferencia significativa $(\mathrm{P}<0.05)$.

El ancho de la grupa anterior (AGa) y posterior (AGp) es $12.1 \pm 0.11$ y $8 \pm 0.13 \mathrm{~cm}$, respectivamente, con un largo de grupa (LG) de $21.4 \pm 0.4 \mathrm{~cm}$; presenta una cabeza dolicocéfala donde el largo (LCa: $20 \pm 0.2 \mathrm{~cm}$ ) es el doble que el ancho de cabeza (ACa: $10.1 \pm 0.27 \mathrm{~cm}$ ); un perímetro de caña (PC) de $7.4 \pm 0.25 \mathrm{~cm}$; en cuanto a la longitud de los cuernos (LC), presenta un dimorfismo (o: $19.7 \mathrm{y}$. $: 12.2 \mathrm{~cm}$ ), la forma de los mismos son opistoceros en forma de sable tipo Aegagrus (58\%) y ortoceros $(37 \%)$.

Según Khandoker et al. (2017), la policromía en una población se debe a cruzamientos no planificados con la introducción de razas diversas sin ningún objetivo productivo definido, en este caso la cabra Chusca lojana presenta una capa policromica con 3 colores básicos (Figura 1), el café en diferentes tonalidades, negro y blanco, solos o combinados en variadas proporciones y lugares del cuerpo; en cuanto a la presencia de mamelas o zarcillos (Carné et al. 2007), el 29\% de cabras Chuscas los poseen, así mismo la presencia de barba, el $26 \%$ de la población la tienen, siendo el biotipo de "oreja torneada" con más predisposición a mamelas y barba, destacando que estas dos características presentan un dimorfismo en favor de los machos.

De acuerdo a lo manifestado por Alía (1996), el perfil frontonasal recto corresponde a cabras que tienen un origen o tronco asiático y las de perfil cóncavo aquellas que tienen un tronco europeo, la mayoría de esta población presenta un perfil de rostro recto (67\%) y subcóncavo (28\%); la forma de la ubre es globosa $(64 \%)$, siendo ideal para evitar golpes y laceraciones en estos suelos irregulares y espinosos, en cuanto a la coloración de pezones y cascos, estos son pigmentados (93 y 87\%, respectivamente). Los chivos Chuscos presentan testículos pendulosos $(70 \%)$, con un diámetro testicular a edad adulta de $30.9 \pm 3.3 \mathrm{~cm}$, una característica peculiar es que el $40 \%$ poseen un escroto dividido en dos sacos (Figura 2).

Al analizar 18 características morfométricas, considerando como variable el sexo (Tabla II), se puede decir que hay un dimorfismo en favor de los machos en el 50\% de las características analizadas $(\mathrm{P}<0,05)$, presentando los mismos una mayor corpulencia y peso, siendo más altos, largos, con mayor fortaleza, capa- cidad torácica y abdominal, una grupa larga, cuernos largos y cañas más gruesas.

\section{DISCUSIÓN}

De acuerdo con el presente estudio se puede manifestar que el peso vivo adulto de esta cabra es ligeramente superior al obtenido por Solis (2017) en otra población de cabras criollas del Ecuador con valores de $37.7 \pm 8.5$ y $53.9 \pm 14.5 \mathrm{~kg}$ en $\rightarrow$ y ${ }^{\lambda}$, respectivamente, o al obtenido por Revidatti et al. (2007) en hembras criollas de Formosa Argentina $(37.87 \pm 6.7 \mathrm{~kg})$. Analizando el largo del cuerpo (LC) la cabra Chusca lojana es longilínea en relación con las cabras nativas de Puebla $(62.8 \pm 1.6 \mathrm{~cm})$ y Oaxaca $(61.6 \pm 6 \mathrm{~cm})$ en México (Hernández et al. 2002, Fuentes et al. 2013, respectivamente), presentando una longitud similar con las cabras de

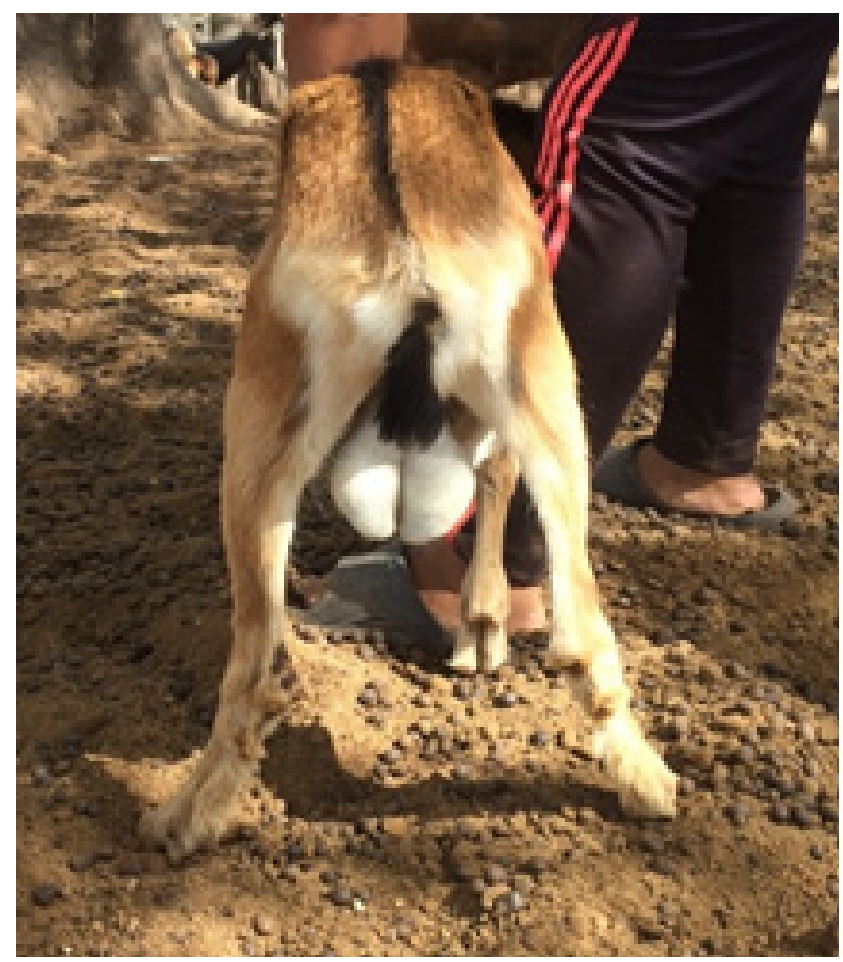

Figura 2. Macho Chusco con saco escrotal partido (Goat buck with split scrotal sac). 
Tabla II. Características morfométricas considerando el sexo en la cabra "Chusca lojana” (cm) (Morphometric characteristics considering sex in the "Chusca lojana" goat).

\begin{tabular}{|c|c|c|c|c|c|c|c|c|c|c|c|c|c|c|c|c|c|c|c|}
\hline Sexo & LC & LCa & LO & $\begin{array}{l}\text { Lcñ } \\
\text { LcñA }\end{array}$ & LcñP & LG & LCu & AP & $\begin{array}{l}A G \\
A G a\end{array}$ & $A G p$ & $A C$ & $\mathrm{AO}$ & PT & PA & PC & DT & ALC & ALG & ADE \\
\hline$\pi$ & $81.2^{\mathrm{a}}$ & 22.4 & 14.2 & 7 & 8 & $24.4^{\mathrm{a}}$ & $19.7^{a}$ & $19.7^{a}$ & 12.6 & 9 & 11.9 & 8.2 & $88.8^{a}$ & $103.4^{\mathrm{a}}$ & $9.4^{\mathrm{a}}$ & 29.9 & $73.9^{a}$ & $74.8^{\mathrm{a}}$ & 33.3 \\
\hline q & $71.7^{b}$ & 20.0 & 12.0 & 6.8 & 7.4 & $21.2^{\mathrm{b}}$ & $12.2^{\mathrm{b}}$ & $15.6^{b}$ & 12.0 & 7.9 & 10.0 & 7.3 & $77.6^{b}$ & $91.6^{b}$ & $7.3^{\mathrm{b}}$ & & $66.5^{\mathrm{b}}$ & $67.1^{\mathrm{b}}$ & 29.8 \\
\hline
\end{tabular}

LC: longitud corporal; Lca: longitud cabeza; LO: longitud oreja; LcñA: longitud caña anterior; LcñP: longitud caña posterior; LG: longitud grupa; Lcu: longitud cuernos; AP: ancho de pecho; AGa: ancho grupa anterior; AGp: ancho grupa posterior; AC: ancho de cabeza; AO: ancho de oreja; PT: perímetro torácico; PA: perímetro abdominal; PC: perímetro caña; DT: diámetro testicular; ALC: altura cruz; ALG: altura grupa; ADE: altura dorso-esternal; ${ }^{*}$ distintas letras en una misma columna: hay diferencia significativa $(P<0.05)$.

la Provincia de Santa Elena-Ecuador (Solis 2017) y de Formosa-Argentina (Revidatti et al. 2007).

La cabra Chusca se la puede considerar como un animal pequeño que presenta una ALC similar a la población estudiada en la Provincia de Santa Elena, siendo más grandes que las cabras mexicanas de Oaxaca $(60.45 \pm 4.9)$, Puebla $(63.7 \pm 1)$ y Formosa en Argentina (62 \pm 3.4$)$. En cuanto al PT es similar al que poseen otras poblaciones criollas de Ecuador (Solis 2017), México (Hernández et al. 2002, Fuentes et al. 2013), Argentina (Revidatti et al. 2007), pero inferior al encontrado en la Blanca Rasquera de España (Carné et al. 2007) y en la cabra Pampeana de Argentina (Bedotti et al. 2004). Presentan un ancho de pecho (AP) similar al que poseen las cabras de México (Hernández et al. 2002, Fuentes et al. 2013) y Argentina (Revidatti et al. 2007), superando a los estándares de las cabras criollas de Santa Elena que se encuentran en ambientes planos y a nivel del mar, pero son inferiores comparándolas con la Blanca Rasquera y Pampeana cuyas medidas en los machos superan los $20 \mathrm{~cm}$. Presenta una ADE más profunda en comparación a las cabras de México (Puebla y Oaxaca) y Formosa en Argentina, pero ligeramente menos profunda que la Blanca Rasquera y la Colorada Pampeana.

Esta cabra posee una grupa estrecha (AGa y AGp), si se la compara con otras poblaciones criollas (Bedotti et al. 2004, Carné et al. 2007, Revidatti et al. 2007, Fuentes et al. 2013, Solis 2017), por lo que es necesario considerar este aspecto en un programa de mejoramiento dada su importancia en la función productiva y reproductiva. La característica LG, presenta al igual que otras poblaciones caprinas un dimorfismo significativo en favor de los machos, la cabra Colorada Pampeana (Bedotti et al. 2004) y de Formosa (Revidatti et al. 2007) presentan similares dimensiones, en tanto que la cabra Blanca Rasquera (Carné et al. 2007), de Puebla y Oaxaca (Hernández et al. 2002, Fuentes et al. 2013) y la cabra de la Península de Santa Elena (Solis 2017), poseen grupas más cortas.

En cuanto a las dimensiones de la cabeza, la cabra Chusca presenta similares medidas que las cabras de Santa Elena, Oaxaca y Formosa, en tanto que la de Puebla presenta una cabeza un poco más corta $(18.2 \pm 0.4$ $\mathrm{cm})$, en este aspecto cabe destacar el gran tamaño que presentan las cabras Rasquera de Cataluña y la Colorada Pampeana de Argentina con medidas que fluctúan entre los 23 y $25 \mathrm{~cm}$ de largo y 12 y $16 \mathrm{~cm}$ de ancho de cabeza. En lo que tiene que ver al PC, es delgado si comparamos con otras poblaciones locales como la Blanca Rasquera $(9.3-10.5 \mathrm{~cm})$, Colorada Pampeana $(8.9-10.9 \mathrm{~cm})$, de Formosa $(8.5 \pm 0.6 \mathrm{~cm})$, de Puebla $(8.4-9 \mathrm{~cm})$ o la cabra de Santa Elena $(8.8-10.9 \mathrm{~cm})$, presentando por tanto esta población una tendencia más a leche que a carne.

La forma y tamaño de las orejas en esta población presentan variabilidad, lo que ha permitido agruparla en 4 biotipos, siendo la cabra Chusca "oreja de león" la que presenta orejas más pequeñas (Long.: 10.7 y ancho: $7.4 \mathrm{~cm}$ ), tamaño similar presenta la Black Bengal de Bangladesh (Jalil et al. 2018), los otros biotipos de la cabra Chusca lojana presentan tamaños (long.: 12.9 y ancho: $7.4 \mathrm{~cm}$ ) similares a la cabra Katjang de Malasia (Khandoker et al. 2017), en general esta población posee orejas pequeñas en comparación a la cabra Borana de Etiopia (Bedada et al. 2019) y a la criolla de Santa Elena-Ecuador (Solis 2017).

En lo que respecta al largo de cuernos (LC) de esta población, un tamaño más corto presentan las cabras Black Bengal de Bangladesh (Jalil et al. 2018) y Andaman de la India (Sunder et al. 2018), la forma de los mismos son similares a los que presentan la criolla de Santa Elena-Ecuador, Colorada Pampeana-Argentina y Andaman-India. Vale destacar que un 22\% de estas cabras son acornes (biotipo Chusca "muca") y de acuerdo al presente estudio los individuos que manifiestan esta característica poseen mayor peso corporal, un porcentaje similar presentó la población criolla de Santa Elena-Ecuador (19\%), y porcentajes inferiores se encontraron en las cabras Blancas de Guerrero-México (7.4\%) y Colorada Pampeana-Argentina (4\%), en tanto que esta característica se manifiesta en el $48 \%$ de las cabras Borana de Etiopia.

En cuanto al color de la capa, similares combinaciones y colores presentan la criolla de Santa ElenaEcuador, Katjang de Malasia, Andaman de India y Oaxaca-México. Otra característica faneroptica es la presencia de mamelas o zarcillos, que según Ricordeau \& Gall (1981), está relacionada con la reproducción y en esta población el porcentaje (29\%), es superior al encontrado en otras poblaciones locales como la cabra de Santa Elena, Colorada Pampeana y Andaman (Solis 2017, Bedotti et al. 2004, Sunder et al. 2018, respectivamente). La presencia de barba en la cabra Chusca es superior a la encontrada en la cabra Andaman (6\%) y Borana (19.8\%), pero inferior a la cabra de Santa Elena. 
Presenta un perfil de rostro similar al de las cabras Andaman de India, Borana de Etiopia, Blanca Rasquera y Blanca Celtiberica de España, Blanca de Guerrero y Puebla en México, Colorada Pampeana y la de Santa Elena-Ecuador. Tienen una forma de ubre globosa, la cual también poseen, pero en un porcentaje menor (51\%) las cabras de Puebla-México, poseen en su gran mayoría pezones y pezuñas pigmentados lo que les proporciona mayor resistencia en estos ambientes, similar característica presentan las cabras Blanca Rasquera de Cataluña y Colorada Pampeana-Argentina.

Los machos Chusco presentan un diámetro testicular superior al que posee el macho Katjang-Malasia $(15.5 \pm 3.3 \mathrm{~cm})$ y Borana-Etiopia $(26.8 \pm 0.6 \mathrm{~cm})$, así mismo la característica de bolsa escrotal partida también la presenta el macho Blanco de Guerrero-México, pero en un porcentaje mayor $(53.8 \%)$. Finalmente el presente estudio también confirmó lo manifestado por Weckerly (1998) y por Polak \& Frynta (2009), que los caprinos pertenecen al grupo de los mamíferos que muestran un mayor dimorfismo sexual en el tamaño corporal, situación que también ha sido encontrada en otras poblaciones locales, como la cabra BoranaEtiopia (Bedada et al. 2019), Black Bengal-Bangladesh (Jalil et al. 2018), Katjang-Malasia (Khandoker et al. 2017), Puebla-México (Hernández et al. 2002), Colorada Pampeana-Argentina (Bedotti et al. 2004) y criolla de Santa Elena-Ecuador (Solis 2017).

\section{CONCLUSIÓN}

La cabra Chusca lojana es un animal elipométrico, longilíneo, con un marcado dimorfismo sexual en el tamaño corporal, longitud de cuernos, perfil frontonasal, presencia de barba y mamelas en favor de los machos, es un animal corto y descarnado con una tendencia productiva más a leche que a carne, destacando su capacidad torácica que le permite recorrer con facilidad por la topografía irregular de la zona en busca de alimento.

\section{AGRADECIMIENTOS}

A la Universidad Nacional de Loja por el financiamiento del Proyecto P05-DI-FARNR; a los Técnicos Veterinarios del MAG-Loja y a los Capricultores de la cabra Chusca lojana del bosque seco de la Provincia de Loja.

\section{BIBLIOGRAFÍA}

Alía, RMJ, 1996 Producción Caprina: La base animal en el ganado caprino, Tomo IX, Prensa M, Madrid.

Bedada, ZE, Gilo, BN, \& Debela, GT, 2019 'Morphometric and Physical Characterization of Borana Indigenous Goats in Southern Oromia, Ethiopia'. Universal Journal of Agricultural Research, vol. 7, $\mathrm{n}^{\circ} .1$, pp. 25-31. http://doi:10.13189/vjar.2019.070104

Bedotti, D, Gómez, A, Sánchez, M, \& Martos, J, 2004 'Caracterización morfológica y faneróptica de la cabra colorada pampeana'. Archivos de Zootecnia, vol. 53, n. 203, pp. 261-271. https://helvia.uco.es/ bitstream/handle/10396/2798/17 1155 03CaracterizacionBe dotti. pdf? sequence $=1$ \&isAllowed $=y$

Bélanger, PJD, 2019 The state of the world's biodiversity for food and agricultura: FAO commission on genetic resources for food and agriculture assessments, FAO, Rome. http://www.fao.org/3/ CA3129EN/ca3129

Carné, S, Roig, N, \& Jordana, J, 2007 'La cabra blanca de Rasquera: caracterización morfológica y faneróptica'. Archivos de Zootecnia, vol. 56, n. 215, pp. 319-330. http://www.redalyc.org/articulo. oa?id $=49521505$

Dubeuf, JP, Morand-Fehr, P, \& Rubino, R, 2004 'Situation, changes and future of goat industry around the world'. Small Ruminant Research, vol. 51, n. 2, pp 165-173. http://doi:10.1016/i.smallrumres.2003.08.007

Falconer, DS, \& Mackay, TF, 1996 Introduction to Quantitative Genetics: Genetics, 4th edition, Addison Wesley Longman, Harlow.

Fuentes, JMS, Alejandre, OM, Chirinos, Z, \& Ricardi, CL, 2013'Zoometría y distribución de partos de la cabra criolla de los valles centrales de Oaxaca'. Actas Iberoamericanas de Conservación Animal, AICA, vol. 3, pp. 150-154. http://www.uco.es/conbiand/aica/templatemo 110 lin photo/articulos/2013/Trabajo022 AICA2013.pdf Hernández, JS, Franco, FJ, Herrera, M, Rodero, E, Sierra AC, Bañuelos, A, \& Delgado, JV, 2020 'Estudio de los recursos genéticos de México: características morfológicas y morfoestructurales de los caprinos nativos de Puebla'. Archivos de Zootecnia, vol. 51, n’. 153-154, pp. 53-64. Jalil, M, Choudhury, M, Kabir, M, \& Habib, M, 2018 'Morphometric characterization of Black Bengal Goat under farming condition in Bangladesh'. Asian Journal of Medical Biology Research, vol. 4, $\mathrm{n}^{\circ}$. 1, pp. 95-104. http://doi:10.3329/ajmbr.v4i1.36827

Khandoker, M, Syafiee, M, \& Rahman, M, 2017 'Morphometric characterization of Katjang goat of Malaysia'. Bangladesh Journal of Animal Science, vol. 45, n. 3, pp.17-24. http://doi:10.3329/ bjas.v45i3.31035

Manson, IL, 1981 'Razas Indígenas de ovinos y caprinos de América Latina': Recursos Genéticos animales en América Latina. Estudio FAOPNUMA: Producción y Sanidad animal 22, pp. 12. http://www.fao. org/3/ah223s/AH223S11.htm

Polák, J, \& Frynta, D, 2009 'Sexual size dimorphism in domestic goats, sheep, and their wild relatives'. Biological Journal of the Linnean Society, vol. 98, n. 4, pp. 872-883.

Revidatti, M, Prieto, P, de la Rosa, S, Capellari, A, Riveiro, M, $2007^{\prime}$ Cabras criollas de la región norte argentina: estudio de variables e índices zoométricos'. Archivos de Zootecnia, vol. 56 n. 1, pp. 479-482.

Ricordeau, G, \& Gall, C, 1981 Genetics: Breeding plans; Goat production, Academic Press, London, RU, pp. 111-169. http://www. sidalc.net/cgi-bin/wxis.exe/? |sisScript=iicacr.xis\&method=post\&fo rmato $=2$ \&cantidad $=1$ \&expresion $=m f n=011806$

Solis, LA, 2017 'Población de cabras de la Provincia de Santa Elena (Ecuador): Su caracterización y pertenencia a los sistemas productivos locales`. Tesis doctoral en Ciencias Agrarias, Facultad de Ciencias Agrarias, Universidad Nacional de Rosario, Argentina, pp 68-102.

Sunder, J, Kundu, A, Kundu, MS, \& Sujatha, T, 2018 'Farming practices and morphometric characterization of Andaman Local Goat'. Indian Journal of Animal Research, article id: B-3615. http://doi:10.18805/ ijar.B-3615

Weckerly, FW, 1998 'Dimorfismo de tamaño sexual: Influencia de los sistemas de masa y apareamiento en los mamíferos más dimorfos'. Journal of Mammalogy, vol. 79, n. 1, pp. 33. https://www.researchgate. net/publication/272152933 Sexual-Size Dimorphism Influence of Mass and Mating_Systems in the Most_Dimorphic_Mammals 